\title{
Phosphoprotein affinity purification identifies proteins involved in S-adenosyl-L-methionine-induced enhancement of antibiotic production in Streptomyces coelicolor
}

\author{
Lingzhu Meng ${ }^{1,3}$, Seung Hwan Yang ${ }^{1,3}$, Sasikumar Arunachalam Palaniyandi ${ }^{1}$, Sung-Kwon Lee ${ }^{1}$, In-Ae Lee ${ }^{1}$, \\ Tae-Jong Kim ${ }^{2}$ and Joo-Won Suh ${ }^{1}$
}

Streptomycetes are the major natural source of clinical antibiotics. The enhanced secondary metabolite production of many streptomycetes by $S$-adenosylmethionine (SAM) in previous studies suggested the existence of a common SAM regulatory effect. We screened nine proteins using the phosphoprotein purification column from Streptomyces coelicolor. Among them, genes (SC05477, SC05113, SC04647, SC04885 and SC01793) for five proteins were disrupted by insertion mutation. The undecylprodigiosin and actinorhodin productions were changed in all mutations. The SAM-induced enhancement of actinorhodin production was abolished by all mutations except SCO4885 mutation, which reduced the production of actinorhodin and undecylprodigiosin with SAM treatment. This study demonstrates that phosphoprotein affinity purification can be used as a screening method to identify the proteins involved SAM signaling.

The Journal of Antibiotics (2011) 64, 97-101; doi:10.1038/ja.2010.148; published online 8 December 2010

Keywords: actinorhodin; protein phosphorylation; S-adenosylmethionine; Streptomyces coelicolor; undecylprodigiosin

\section{INTRODUCTION}

Streptomyces is the major genus that produces useful antibiotics of natural origin. Secondary metabolite production in Streptomyces is usually strain specific; thus, it is difficult to develop a universal method to promote the production of secondary metabolites in most Streptomyces strains. ${ }^{1,2}$ We observed that increased concentration of $S$-adenosylmethionine (SAM) by overexpression of SAM synthetase gene stimulated actinorhodin production in Streptomyces lividans, ${ }^{3,4}$ and exogenous SAM treatment in culture media enhanced streptomycin production in S. griseus, ${ }^{5}$ as well as other secondary metabolite production in many Streptomyces strains. ${ }^{6}$ On the basis of these results, we sought to identify SAM-response proteins, which might be targets, to improve secondary metabolite production in many Streptomyces spp.

SAM-enhanced secondary metabolite production in all tested actinomycetes suggested the existence of a common signal transduction pathway. To identify cellular proteins that regulate the SAM response, we hypothesized that changes in protein phosphorylation constitute part of the signal transduction of SAM responses in S. coelicolor, a model strain for actinomycetes. As a new approach to identify proteins that regulate signal transduction on treatment with SAM, a phosphoprotein purification method was adopted to find proteins whose characteristics were altered by SAM. We identified five proteins, and their effects on antibiotic production were evaluated in this study.

\section{MATERIALS AND METHODS}

Bacterial strains and culture conditions

S. coelicolor M130 was used in this study. Escherichia coli $\mathrm{DH} 5 \alpha \mathrm{F}^{\prime}$ and E. coli ET12567 were used as hosts for routine subcloning and transformation, respectively. $\mathrm{R}^{-}$medium, which was modified from $\mathrm{R} 5$ medium to lack $\mathrm{KH}_{2} \mathrm{PO}_{4}, \mathrm{CaCl}_{2}$ and L-proline, was used for culturing and measuring antibiotic production in $\mathrm{M} 130 .^{7}$ YEME medium was used for protoplast regeneration of M130. ${ }^{8}$ Mycelial growth, spore and protoplast preparations and transformation of M130 were performed as described previously. ${ }^{7}$ To ensure reproducibility, all transformations were performed with freshly prepared protoplasts from the same batch of mycelium, and all transformations were repeated at least three times. If necessary, ampicillin, apramycin or neomycin was added to the culture medium at $50 \mu \mathrm{g} \mathrm{ml}^{-1}$, thiostrepton was used at $5 \mu \mathrm{g} \mathrm{ml}^{-1}$. SAM was added

\footnotetext{
${ }^{1}$ Division of Bioscience and Bioinformatics, College of Natural Science, Myongii University, Gyeonggi-Do, Korea and ${ }^{2}$ Department of Forest Products and Biotechnology, College of Forest Science, Kookmin University, Seoul, Korea

${ }^{3}$ These authors contributed equally to this work.

Correspondence: Professor T-J Kim, Department of Forest Products and Biotechnology, College of Forest Science, Kookmin University, Seongbuk-gu, Seoul 136-702, Korea. E-mail: bigbell@kookmin.ac.kr or

Professor J-W Suh, Division of Bioscience and Bioinformatics, College of Natural Science, Myongji University, Cheoin-gu, Yongin, Gyeonggi-Do 449-728, Korea.

E-mail: jwsuh@mju.ac.kr

Dedicated to late $\operatorname{Dr} \mathrm{C}$ Richard Hutchinson for his exceptional contributions to natural product biosynthesis, engineering and drug discovery.

Received 30 September 2010; revised 1 November 2010; accepted 3 November 2010; published online 8 December 2010
} 
to the medium to a final concentration of $10 \mu \mathrm{M}$ at $12 \mathrm{~h}$ of culture after inoculation.

\section{Purification and identification of proteins}

S. coelicolor M130 was cultured in $\mathrm{R}^{-}$liquid medium at $28^{\circ} \mathrm{C}$ and 250 r.p.m. Cells were collected from the culture, with and without SAM treatment, after $12 \mathrm{~h} \mathrm{SAM}$ treatment. Cells were suspended in lysis buffer ( $10 \mathrm{~mm}$ Tris- $\mathrm{HCl}, \mathrm{pH}$ 8.0, 1 mм EDTA, 10\% glycerol and $100 \mu$ sodium vanadate) and disrupted by sonication (Sonifier 450, Branson Ultrasonics, Danbury, CT, USA). The cell debris were discarded and proteins were purified using the PhosphoProtein Purification Kit according to the manufacturer's instructions (Qiagen, Seoul, Korea). Eluted proteins were separated by $10 \%$ SDS-PAGE gel and visualized with Coomassie blue. To identify the separated proteins, individual bands were excised from the gel and analyzed by MS (Supplementary methods) and compared with a S. coelicolor proteomic database based on the genomic database (http://www.sanger.ac.uk). The search results are shown in Supplementary Table S1.

\section{Disruption of genes corresponding to the identified proteins}

To prepare plasmids for gene disruption, an internal DNA fragment of each target gene (SCO5477-1006bp; SCO5113-1018bp; SCO4647-752 bp; SCO4885-801 bp; SCO1793-462 bp) was amplified by PCR from M130 chromosomal DNA using the primer pairs that are listed in Table 1. PCR products were cloned into pOJ260 using XbaI and HindIII restriction enzymes and transformed into DH5 $\alpha \mathrm{F}^{\prime}$. Purified plasmids from $\mathrm{DH} 5 \alpha \mathrm{F}^{\prime}$ were re-transformed into ET12567. After another round of purification, the plasmids were transformed into M130 according to previous methods. ${ }^{7}$ Apramycin-resistant candidates were selected, and each mutant was confirmed by PCR reactions using primers listed in Table 1. The two primer pairs consisted of POJ260-C,

Table 1 List of primers

\begin{tabular}{|c|c|c|}
\hline Name & Target gene & Primer \\
\hline \multicolumn{3}{|c|}{ Primers for gene disruptions } \\
\hline SC05113-A & Sc05113 & 5'-CAATCTAGAAGCATTCTCCGTAACCGCAC-3' \\
\hline Sc05113-B & Sc05113 & 5'-CAAAAGCTTTACATCCGCTCGATCGTGTG-3' \\
\hline SC05477-A & Sc05477 & 5'-CAATCTAGAACTACCCAACGCACCTCAG-3' \\
\hline Sc05477-B & Sc05477 & 5'-CAAAAGCTTCACCTCCTTGAGGTACGTC-3' \\
\hline SC04647-A & Sc04647 & 5'-CAATCTAGATGACCCGAACGTGAACGAC-3' \\
\hline SC04647-B & Sc04647 & 5'-CAAAAGCTTTTCTGGCGGATCGTCTTGC-3' \\
\hline SC04885-A & SC04885 & 5'-CAATCTAGACCGGATTTCCCGGATCAC-3' \\
\hline SC04885-B & Sc04885 & 5'-CAAAAGCTTGACCGTGTTGGTCTTGGTG-3' \\
\hline SC01793-A & Sc01793 & 5'-CAATCTAGAGCGTTCAAGAAGCTGCTCG-3' \\
\hline SCO1793-B & SCO1793 & 5'-CAAAAGCTTCATGTCCGCGCTCTTGAAG-3' \\
\hline \multicolumn{3}{|c|}{ Primers for the confirmation of gene disruptions } \\
\hline POJ260-C & pOJ260 & 5'-GAATTCGATATCGCGCGC-3' \\
\hline Sc05113-C & Sc05113 & 5'-GAATTCGATATCGCGCGC-3' \\
\hline SC05477-C & SC05477 & 5'-CACGAAGTCGTACATACCG-3' \\
\hline SC04647-C & Sc04647 & 5'-TGGTCGAAGCTCAGCTC-3' \\
\hline SC04885-C & Sc04885 & 5'-GCTGCTCTTGACCTTGATC-3' \\
\hline SCO1793-C & SCO1793 & 5'-AGAACCAGTTGCGCTTGC-3' \\
\hline \multicolumn{3}{|c|}{ Primers for the construction of complemented plasmids } \\
\hline SC05113-D & Sc05113 & 5'-CAAGAATTCAAGGGGAACCCCCCACATC-3' \\
\hline SC05113-E & Sc05113 & 5'-CAAGGATCCTTACTTCTTGAGGAAGACCCGG-3' \\
\hline SC05477-D & Sc05477 & 5'-CAAGAATTCCCCTGCCGTACCGCAAAG-3' \\
\hline SC05477-E & Sc05477 & 5'-CAAGGATCCTTACTTCTTGGGGTTCTTCAGAC-3' \\
\hline SC04647-D & Sc04647 & 5'-CAAGAATTCGTCGAGAGCGAAGGGCG-3' \\
\hline SC04647-E & Sc04647 & 5'-CAAGGATCCCTAGTTCTTCTGGATCTGGTCG-3' \\
\hline SCO4885-D & Sc04885 & 5'-CAAGAATTCGCACACACCCGCACGAAG-3' \\
\hline SCO4885-E & SC04885 & 5'-CAAGGATCCTCAGCTGCTCTTGACCTTGATC-3' \\
\hline SCO1793-D & Sc01793 & 5'-CAAGAATTCTGCCGTCCGAGCGTAACG-3' \\
\hline SCO1793-E & SCO1793 & 5'-CAATCTAGACTAGAACCAGTTGCGCTTGC-3' \\
\hline
\end{tabular}

which was the outside region of the inserted fragments on pOJ260 for one end, and the corresponding primer of each gene, which was the outside region of the target genes for PCR.

\section{Complementation of genes}

Entire open reading frames of the target genes were amplified by PCR from S. coelicolor chromosomal DNA using the primer pairs listed in Table 1. The PCR products were cloned under the ermE promoter of pWHM3 using EcoRI and $B a m H I$ restriction sites and transformed into DH5 $\alpha \mathrm{F}^{\prime}$. Ampicillin-resistant candidates were selected, and the cloned genes were confirmed by sequencing. After another transformation into ET12567, the plasmids were transformed into M130. The complemented strains were selected on the basis of thiostrepton resistance.

\section{Quantification of actinorhodin and undecylprodigiosin}

S. coelicolor M130 was grown for $48 \mathrm{~h}$ in $\mathrm{R} 5^{-}$liquid medium at $28^{\circ} \mathrm{C}$ and 250 r.p.m. Further, the cells were subcultured to $1 \%$ (final volume) in fresh $\mathrm{R}^{-}$liquid medium, and grown at $28^{\circ} \mathrm{C}$ and 250 r.p.m. for the indicated times in the text and figures. Cell growth measurements and quantitative analysis of actinorhodin and undecylprodigiosin were performed as described previously. ${ }^{9}$

\section{RESULTS}

\section{Identification of proteins involved in SAM signaling}

To identify proteins involved in SAM signaling, we prepared total protein from $S$. coelicolor, with or without SAM treatment, and resolved on 10\% SDS-PAGE gel. No obvious difference in the protein profile was observed between SAM-treated and control samples (data not shown), however, purification of cell extracts with a phosphoprotein binding column showed clear differences in protein profile between samples, with or without SAM treatment (Figure 1). On the basis of the comparison of protein bands in the eluate obtained from samples with or without SAM treatment, 10 regions were selected (Figure 1), and the bands were excised from the gel and the protein composition of those bands was analyzed by MS, followed by identification of proteins using MS/MS spectra by searching the proteomic database of $S$. coelicolor. The A1 and A2 regions in Figure 1 were excised as one piece, and their protein content was determined together. The B2 and B3 regions in Figure 1 were determined similar to A1 and A2 regions. The detailed MS results are shown in Supplementary Table S1. The identified proteins were listed in Table 2. The A1 and A2 regions had oligopeptide-binding lipoprotein (SCO5477), cell division trigger factor (SCO2620) and ABC transport system lipoprotein (BldKB, SCO5113). Notably, oligopeptide-binding lipoprotein (SCO5477) and ABC transport system lipoprotein (BldKB, SCO5113) were also identified in protein bands present in $\mathrm{B} 2$ and $\mathrm{B} 3$. The $\mathrm{A} 3$ region contained an antitermination protein (NusG, SCO4647) for transcription. The A4 and A5 regions contained a sugar-binding protein (SCO4885) for transport and a putative sporulation control protein (Spo0M, SCO1793), respectively. The B1 region contained the RNA polymerase beta prime subunit (RpoC, SCO4655). The B4 and B5 regions contained glutamine synthetase (SCO2210) and superoxide dismutase (SCO2633), respectively. As the biological functions of RNA polymerase beta prime subunit, glutamine synthetase and superoxide dismutase were known, ${ }^{10}$ they were excluded from further examination. Cell division trigger factor was also excluded in this study because of a possible requirement for cell growth. ${ }^{10,11}$

\section{Changes in growth and antibiotic production by disruption of the identified genes}

To further analyze the link between the identified proteins and the SAM effect, the corresponding genes (SCO5477, SCO5113, SCO4647, SCO4885 and SCO1793) were disrupted by inserting a fragment of 
DNA internal to each target gene amplified from S. coelicolor genomic DNA. Each mutant strain was analyzed for undecylprodigiosin and actinorhodin production, with or without $10 \mu \mathrm{M}$ SAM treatment. The SCO5477 mutant lost nearly all of its responsiveness to SAM without any change in undecylprodigiosin production (Figure 2a). The SCO5113 and SCO4647 mutants also lost SAM responsiveness,

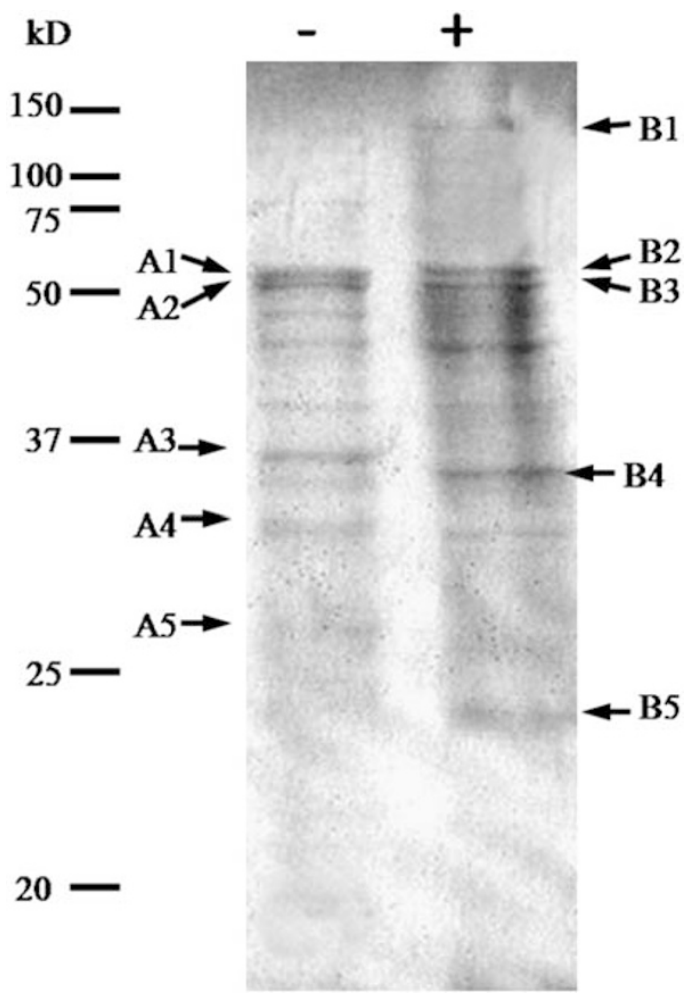

Figure 1 SDS-PAGE separation of proteins, purified by phospho-affinity. Cell extracts were made by rupturing $S$. coelicolor M130 cells followed by centrifugation. After binding proteins in the phosphoprotein purification column, proteins in the eluate were separated by electrophoresis on a $10 \%$ SDS-PAGE gel. Marking on the left (A1-A5) and right (B1-B5) indicate the regions excised for MS analysis. The symbols '-' and ' + ' indicate no treatment and $10 \mu \mathrm{m}$ SAM treatment, respectively. The sizes of marker proteins are shown on the left in kilodalton $(\mathrm{kDa})$. The identification results of the regions are summarized in Table 2. but their production of undecylprodigiosin was higher than wild type (Figures $2 \mathrm{~b}$ and c). On treatment with SAM, the SCO4885 mutant showed low level of undecylprodigiosin production-the opposite response of the wild type to SAM (Figure 2d). The SCO1793 mutant retained its response to SAM, but its overall production of undecylprodigiosin decreased dramatically (Figure 2e). The complemented strains for all five mutations were constructed by introducing the entire gene to the mutant strain, and all restored the level of undecylprodigiosin production and responsiveness to SAM (Supplementary Figure S1), which indicates the change in undecylprodigiosin production, was because of the target gene disruption.

In the case of actinorhodin production, all mutants except SCO4885, lost the SAM responses (Figure 3). The SCO4885 mutant showed reduced actinorhodin production on treatment with SAM (Figure 3d). This phenomenon was also observed in undecylprodigiosin production, as shown in Figure 2d. Actinorhodin production increased significantly in the SCO5477 mutant. Actinorhodin production was similar to wild-type levels, and the response to SAM was restored in all of the complemented strains (Supplementary Figure S2).

\section{DISCUSSION}

The use of a phosphoprotein purification method in this study enhanced protein concentrations specifically, and nine proteins were identified as the dominant proteins in the selected band regions, based on the comparison before and after SAM treatment. Among them, mutations in the five candidate genes had unique effects on antibiotic production and the SAM response. Furthermore, two of the five proteins, oligopeptide-binding lipoprotein (SCO5477) and ABC transport system lipoprotein (BldKB, SCO5113), had also been observed in a previous study related to SAM-induced antibiotic production. ${ }^{12}$

Of the five candidate genes, none completely abolished the production of undecylprodigiosin or actinorhodin, suggesting that they are dispensable for undecylprodigiosin and actinorhodin synthesis. But the undecylprodigiosin production was enhanced by the SCO5113 and SCO4647 mutations and reduced by the SCO5477, SCO4885 and SCO1793 mutations, implicating their involvement in undecylprodigiosin production (Figure 2). The enhancement by SCO5477 and SCO4885, and a reduction by the SCO1793 mutation also demonstrate their regulation of actinorhodin synthesis (Figure 3). Because any mutation that can change cell physiology is a potential candidate

Table 2 Identified proteins from phospho-affinity purification before and after SAM treatment

\begin{tabular}{|c|c|c|c|c|c|}
\hline Location $^{\mathrm{a}}$ & Gene & Suggested function & Protein & Total peptides ${ }^{\mathrm{b}}$ & $\mathrm{M}_{W}(k D a)^{\mathrm{c}}$ \\
\hline $\mathrm{A} 1$ and $\mathrm{A} 2$ & Sc05477 & Oligopeptide-binding lipoprotein & SC2A11.11 & 8 & 65.27 \\
\hline $\mathrm{A} 1$ and $\mathrm{A} 2$ & SC02620 & Cell division trigger factor & ScC80.05c & 5 & 51.13 \\
\hline $\mathrm{A} 1$ and $\mathrm{A} 2$ & Sc05113 & BIdKB, ABC transport system lipoprotein & SCBAC31E11.09 & 4 & 65.49 \\
\hline A3 & Sc04647 & NusG, transcription antitermination protein & SCD82.18 & 4 & 32.82 \\
\hline A4 & SC04885 & Putative lipoprotein & 2SCK8.11 & 1 & 36.04 \\
\hline A5 & SC01793 & SpoOM, putative sporulation-control protein & $\mathrm{SCI} 5.01$ & 2 & 28.44 \\
\hline $\mathrm{B} 1$ and $\mathrm{B} 2$ & SC04655 & RpoC, DNA-directed RNA polymerase beta' chain & SCD82.27 & 2 & 144.51 \\
\hline $\mathrm{B} 1$ and $\mathrm{B} 2$ & Sc05113 & BIdKB, ABC transport system lipoprotein & SCBAC31E11.09 & 4 & 65.49 \\
\hline B3 & Sc05477 & Oligopeptide-binding lipoprotein & SC2A11.11 & 3 & 65.27 \\
\hline B4 & SCO2210 & Glutamine synthetase & SC10B7.05 & 10 & 37.16 \\
\hline B5 & SC02633 & Superoxide dismutase & SC8E4A.03 & 4 & 23.51 \\
\hline
\end{tabular}

aBand number indicated on the gel of Figure 1.

bTotal number of peptides used for comparison with the database.

cMolecular weight $\left(M_{\mathrm{W}}\right)$ of the proteins identified from the database. 

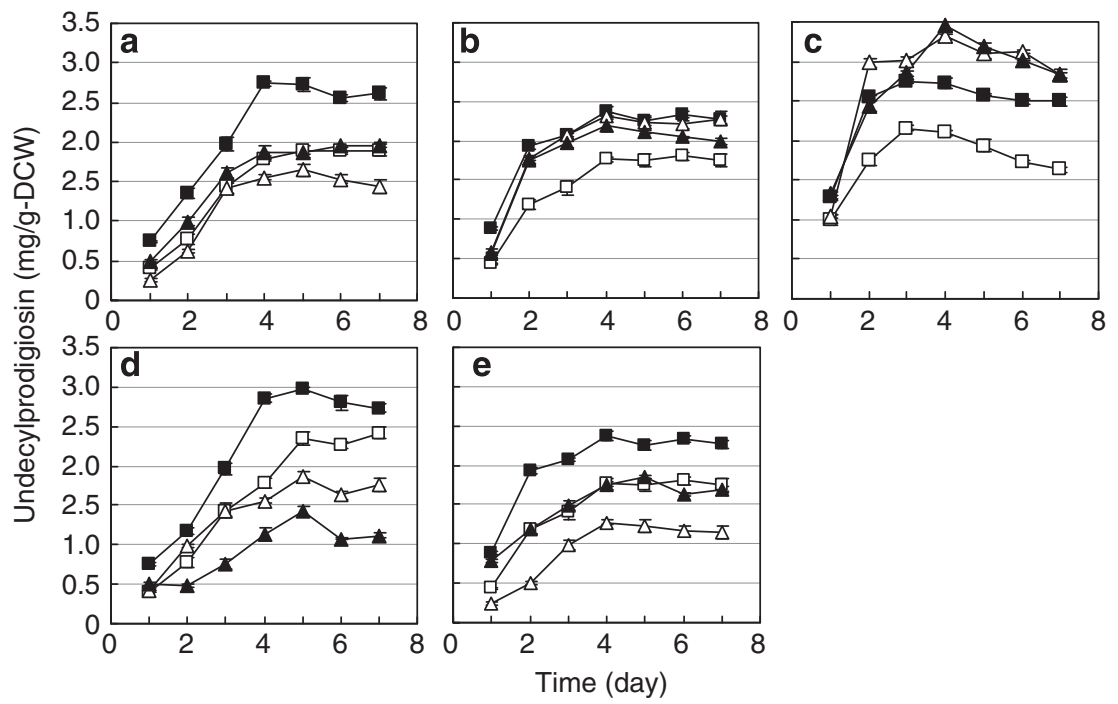

Figure 2 The undecylprodigiosin production of mutants. At the indicated times, the concentration of undecylprodigiosin in the broth was measured and divided by the dry cell weight (DCW) to determine the specific amounts of production. $\square$, wild-type; $\mathbf{\square}$, wild-type with $10 \mu \mathrm{M}$ SAM; $\triangle$, mutant; $\mathbf{\Delta}$, mutant with $10 \mu \mathrm{M}$ SAM. The mutated genes are SC05477 in (a), SC05113 in (b), SCO4647 in (c), SCO4885 in (d) and SCO1793 in (e).
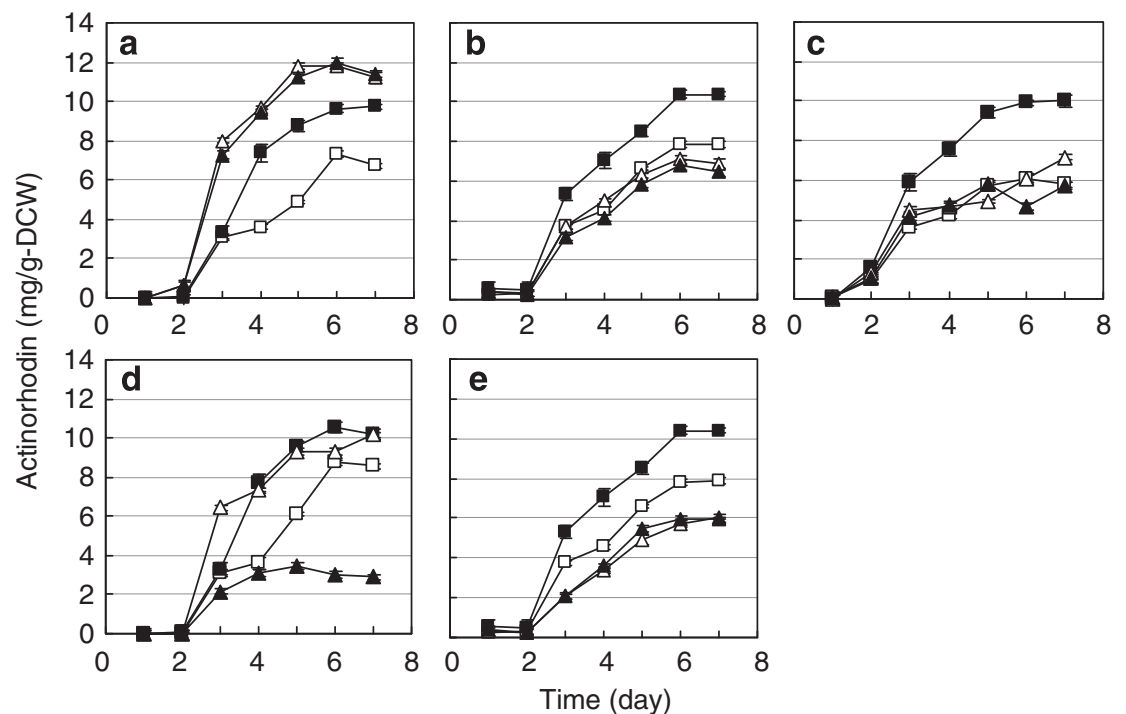

Figure 3 The actinorhodin production of mutants. At the indicated times, the concentration of actinorhodin in broth was measured and divided by the dry cell weight to determine the specific amounts of production. $\square$, wild-type; $\mathbf{\square}$, wild-type with $10 \mu \mathrm{M} \mathrm{SAM;} \triangle$, mutant; $\mathbf{\Delta}$, mutant with $10 \mu \mathrm{M}$ SAM. The mutated genes are SC05477 in (a), SC05113 in (b), SC04647 in (c), SCO4885 in (d) and SCO1793 in (e).

affecting secondary metabolite production, further well-designed experiments are required to decide whether each mutation, directly or indirectly, affects antibiotic production and to elucidate the functional mechanism in cells.

However, the response to SAM in undecylprodigiosin production was abolished by the SCO5477, SCO5113 and SCO4647 mutations, and for all five mutations except SCO4885, the enhancement of actinorhodin production by SAM was abolished. Interestingly, the addition of SAM reduced the production of actinorhodin and undecylprodigiosin in the SCO4885 mutant. This result is the first observation of decreasing secondary metabolite production after treatment with SAM.

This study demonstrated that the phosphoprotein affinity purification column could be applied successfully to search for proteins, whose phosphorylation status is altered on SAM treatment, and suggest that the five identified proteins (SCO5477, SCO5113, SCO4647, SCO4885 and SCO1793) regulated secondary metabolite production and SAM-induced enhancement with their own specific effect.

\section{ACKNOWLEDGEMENTS}

This work was supported by a grant (11-2008-16-001-00) from the 21C Frontier Microbial Genomics and Application Center program, the Korean Ministry of Science and Technology and by a National Research Foundation of Korea (NRF) grant, funded by the Korean government (MEST) (Grant\#: 20090071562). Lingzhu Meng is supported by the Korea Research Foundation Grant funded by the Korean Government (MOEHRD) (KRF-2007-211-C00039) and the second stage of the Brain Korea 21 (BK21) Project. 
1 Huang, J. et al. Cross-regulation among disparate antibiotic biosynthetic pathways of Streptomyces coelicolor. Mol. Microbiol. 58, 1276-1287 (2005).

2 Novotna, J. et al. Proteomic studies of diauxic lag in the differentiating prokaryote Streptomyces coelicolor reveal a regulatory network of stress-induced proteins and central metabolic enzymes. Mol. Microbiol. 48, 1289-1303 (2003).

$3 \mathrm{Kim}, \mathrm{D}$. J. et al. Accumulation of S-adenosyl-L-methionine enhances production of actinorhodin but inhibits sporulation in Streptomyces lividans TK23. J. Bacteriol. 185, 592-600 (2003).

4 Okamoto, S., Lezhava, A., Hosaka, T., Okamoto-Hosoya, Y. \& Ochi, K. Enhanced expression of $S$-adenosylmethionine synthetase causes overproduction of actinorhodin in Streptomyces coelicolor A3(2). J. Bacteriol. 185, 601-609 (2003).

5 Shin, S.K., Xu, D., Kwon, H. J. \& Suh, J. W. S-adenosylmethionine activates adpA transcription and promotes streptomycin biosynthesis in Streptomyces griseus. FEMS Microbiol. Lett. 259, 53-59 (2006).

6 Huh, J. H. et al. Widespread activation of antibiotic biosynthesis by S-adenosylmethionine in streptomycetes. FEMS Microbiol. Lett. 238, 439-447 (2004).
7 Kieser, T., Bibb, M. J., Buttner, M. J., Chater, K. F. \& Hopwood, D. A. Practical Streptomyces genetics (John Innes Foundation, Norwich, United Kingdom, 2000).

8 Hopwood, D. A. et al. Genetic Manipulation of Streptomyces. A Laboratory Manual (John Innes Foundation, Norwich, 1985).

9 Li, M., Kim, T. J., Kwon, H. J. \& Suh, J. W. Effects of extracellular ATP on the physiology of Streptomyces coelicolor A3(2). FEMS Microbiol. Lett. 286, 24-31 (2008).

10 Park, S. S. et al. Mass spectrometric screening of transcriptional regulators involved in antibiotic biosynthesis in Streptomyces coelicolor A3(2). J. Ind. Microbiol. Biotechnol. 36, 1073-1083 (2009).

11 D'Alia, D., Nieselt, K., Steigele, S., Muller, J., Verburg, I. \& Takano, E. Noncoding RNA of glutamine synthetase I modulates antibiotic production in Streptomyces coelicolor A3(2). J. Bacteriol. 192, 1160-1164 (2010).

12 Park, H. S., Shin, S. K., Yang, Y. Y., Kwon, H. J. \& Suh, J. W. Accumulation of $S$-adenosylmethionine induced oligopeptide transporters including BldK to regulate differentiation events in Streptomyces coelicolor M145. FEMS Microbiol. Lett. 249, 199-206 (2005).

Supplementary Information accompanies the paper on The Journal of Antibiotics website (http://www.nature.com/ja) 